\title{
Optimal Content Downloading in Vehicular Networks
}

\author{
K.Vishal ${ }^{1}$, Hanuman Prasad ${ }^{2}$ \\ ${ }^{I}$ Student/M. Tech, AMR Institute of TechnologyAdilabad, \\ ${ }^{2}$ Associate professor/IT HOD, AMR Institute of Technology Adilabad.
}

\begin{abstract}
We consider a system where users aboardcommunication-enabled vehicles are interested in downloading different contents from Internet-based servers. This scenario captures many of the infotainment services that vehicular communication is envisioned to enable, including news reporting, navigation maps and software updating, or multimedia file downloading. In this paper, we outline the performance limits of such a vehicular content downloading system by modeling the downloading process as an optimization problem, and maximizing the overall system throughput. Our approach allows us to investigate the impact of different factors, such as the roadside infrastructure deployment, the vehicle-to-vehicle relaying, and the penetration rate of the communication technology, even in presence of large instances of the problem. Results highlight the existence of two operational regimes at different penetration rates and the importance of an efficient, yet 2-hop constrained, vehicle-to-vehicle relaying.
\end{abstract}

Keywords: Vehicular network, Content downloading Process,Max-flowproblem, Optimization, Density measurement.

\section{Introduction}

Thecommunication-enabledvehiclesare interested in downloading differentmultimediacontents fromInternet-basedservers. Thissystem capturesmanyoftheentertainmentserviceswith effectiveinformation,such asnavigationmaps,newsreportingservice, andsoftwareupdating, or multimediacontentdownloading. Inthisapproach bothinfrastructure-to-vehicleandvehicle-toThemajoraimistomaximizetheoverallsystemthroughput; weformulateamax-flowproblem thataccountsfor severalpracticalaspects,suchaschannel contention and the datatransferparadigm.

Asaresult,Multimediacontentdownloading invehicularnetworksby thevehicleshasreceived increasingattention from theresearchcommunity.Initially,theavailabilityofInfrastructure-to- Vehicle (I2V)communication capabilitiesare based on high-throughputDedicated Short-Range Communication(DSRC)technologies, isseenasanopportunityfor transferoflargedatato mobilenodesthatwouldnotbepossiblethroughtheexisting $2 \mathrm{G} / 3 \mathrm{G}$ infrastructure,Nextthe availabilityofVehicle-toVehicle (V2V)connectivityhas fostered anumberofproposals to makeuseofthecooperation among vehicularusers soasto improvetheirdownloadingperformance.In particular, V2Vconnectivitybasedapproachesareespecially goodwhenoneconsiders thatthe infrastructurecoverage willbe mottled atinitialstages, and barelyseamless even at laterones.

Previousworksoncontentdownloading invehicularnetworkshavedealtwithindividualaspects of the process,such as roadsideAPs deployment, the performanceevaluation ofl2Vcommunication,thenetwork connectivity,ofV2Vdatatransferparadigms.Noonehastriedto dealwiththeproblem asawhole, trying toquantify theactualpotentialofanI2V/V2V-based fillsuchagap,weintroducethefollowing questions:(i) whichisthemaximumdownloadingperformance achievablethroughDSRC-basedI2V/I2 V communication, inagiven mobilityscenario?(ii) Whataretheimportantfactorsthatmainly determinesucha downloadingperformance?

To answer thesequestions, wecombinethisdownloading process toa mixedinteger linear programming (MILP)knownasmaxflowproblem.Thesolutionthisproblem resultsinthe optimalAccess pointdeploymentoveragivenroad layoutandany possible combinationof $\mathrm{V} 2 \mathrm{~V}$ and $\mathrm{I} 2 \mathrm{Vdata}$ transmission.

Ourframework introducesaDTNG time-invariantgraph.Wedonotundertakethecontacts betweenmobilenodestobesamebutallowthemtoaccessdirectly,andalsoreportthepresence

ofroadsideinfrastructureandchannelcontention.Suchanapproachallowsus tosignificantly enhance the AP deployment over the given road layout, since we maximize the overall throughputandalsoprovide theoptimalsolutioninstead ofan approximation.

Atthe result, theaccesspointorrelayshows thevehiclecapability priorandsendsthe corresponding lowqualityorhighquality file.Thisachievesthevehicle toreceivetheproperfile resource.Vehicledensityiscalculatedbasedonprevioustemporalchangesand thenewvehicle density iscalculated. The accesspoints'capabilitiesareadjusted sothat itworksmoreinhigh vehicledensityenvironmentand works less in lowvehicle densityenvironment. 
Thispaperisorganizedasfollows:SectionII

describesthepreviouswork, whileSectionIII discussescontribution ofwork.In Section IV, webuild thesystem modelandassumption, while wegenerate theDynamic Network topology graphin Section Vandwe formulate themax-flow problem inSection VI,Results, derivedinthedesignguidelinesdescribed inSection VII.In sectionVIII, weevaluatethevehicledensitybaseddata downloading.SectionIXdescribes Security issues;finally section Xsummarizesourmajorfindingsandpointoutdirection offuture work.

\section{Releted Work}

TheauthorsU.Paul,M.M.Buddhikot,A.P.Subramanian,andS.R.Daswerestatedthat completemeasurementanalysisofnetworkresourcedeploymentandthesubscriberactivities scaledatasetcollectedwithinanationwide3 Gcellularnetwork.Thedatasetkeeps

the closetomorenumberofsubscribersoverthousands ofbasestations. They alsoexaminethe capability of network resourceswhichcan beused by differentsubscribersaswellasby different applications. They also find outthe traffic in vehicularnetwork fromthe pointofviewof thebase stations and analyze the temporal and spatial variations in different kinds of the vehicularnetwork.Inorder toaddresssuchcoverageuncertaintiestheauthorsZ.Zheng,P.Sinha, andS. Kumarweregivenaidea aboutnewthealternating coverageformobileusers,called $\alpha$-coverage, andexaminedhowsuchcoveragecanbeattainedbysystematicdeploymentofmoreAPsto createanefficiently scalableinfrastructure. Inotherway,adeploymentofAPsinvolvedin $\alpha$ -

coveragetoanetworktopology,iftheroadwithlengthoonthegivennetworkresourcemeets withatleastoneAPinthat resource.TheauthorsZ.Lu,Z.Zheng,P.Sinha,and S.Kumarwere alsostated thatwith increasing popularity ofmediaenableddevices; theneed forhighdata-rate servicesformobileusers isobvious.Large-scale WirelessLANs(WLANS)canoffersuch a service, but theyareveryexpensive to deployandmaintain. The above resultsnotmake the grade to provide anythroughputassuranceto a vehicularuser; itcan onlyprovide opportunistic services to them.

\section{My Contribution}

Thedensitymeasurementin vehicularnetworkmycontributions to this problemare as follow:

Theaccesspointorrelay tracksthevehiclecapability priorandsendsthecorresponding low qualityorhigh qualityfile. This achieves the vehicletoreceive the properfileresource

Vehicledensityiscalculatedbasedonprevioustemporalchangesandthenewvehicle densityiscalculated.

$\square$ Theaccesspoints'capabilitiesareadjustedsothatitworksmoreinhighvehicledensity environmentand works less inlowvehicle densityenvironment.

$\square$ Vehicle densitybased downloadscenariois appliedtoAccessPoints.

ProposedmethodswheretheRoadside infrastructure i.e.,accesspointsareworking atdifferent capabilities irrespective ofvehicledensity.

\subsection{NetworkModel}

\section{Systemmodel And Assumptions}

WecreateanetworkcomposedoffixedroadsideAPsandvehicularusers, wheresomeofthem aredownloader's. Theyareinterestedin downloadingmultimediacontent fromtheInternet through theAPs.Weconsider thegeneralcase inwhichevery downloadermaybeinterestedin different content. They can either use relays or establish direct connectivity with APs. In particular, we consider thefollowingdata transfer paradigms:

Directtransfers,adirectcommunicationbetweenanAPandadownloader.Thisshowsthe typicalwayhow the mobileuserscommunicatewiththe infrastructureasin today'swireless networks;

Connectedforwarding,the resultshowscommunicationmadethroughoneormorevehicles that createamultihoppathbetweenanAPand adownloader. This is theconventionalapproach to traffic deliveryin ad hocnetworks;

Carry-and-forward,thecommunicationmadethroughoneormorevehiclesthatstoreandcarry thedata,anddelivering them eithertothetargetdownloaderortoanotherrelaywhichmeetsuch downloadersooner. 
Ourapproach allowsustoprocessing aroad layoutandanassociatedvehicularmobility trace,so asto builda timeexpandedgraphthat represents the temporalnetwork evolution(Sec.V).By using thisgraph,weformulateamaxflowproblem; thesolutionofthis problemmatchesour goals.

\section{Dynamicnetwork Topologygraph}

Dynamicnetwork topologygraph(DNTG)generateafrom adifferentvehicularmobility tracein network topology,considering thatonthecorresponding roadlayoutthereare:(i)asetofA candidatelocations( $, \mathrm{i}=1, \ldots, \mathrm{A})$ whereAPscouldbeplaced(ii)asetofVvehicles( $, \mathrm{i}=1, \ldots, \mathrm{V})$ travelovertheroadlayout(iii)asetofDvehiclesthatwishtodownloaddatafromtheAPs.

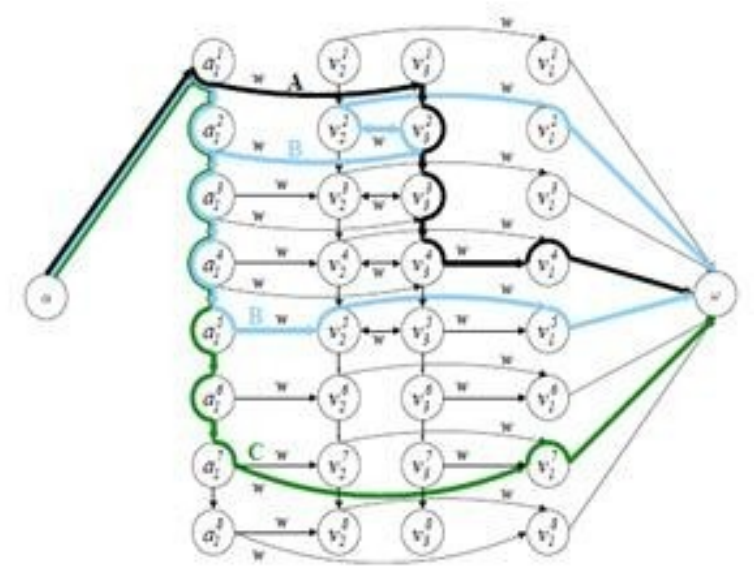

Figure1.AsampleDNTG, withone AccesspointAandthreevehiclesv1,v2\&v3, thevehicle(v1)isa downloaderwhiletheothers(v2,v3)canactasrelays.Intheabovegraph,weshowuppathsthatareagent ofthecarryandforward(A), connectedforwarding(B), anddirecttransfer(C)paradigms.

Themajoraim ofthistopologygraphistomodelallpossiblewaysthroughwhichdatacanflow eitherdirectAPstothedownloader'sorpossiblyviarelays. Withknownmobility

from identifythecontacteventsbetween anypairofnodes such asV2I/V2V.

trace,we

Each contacteventischaracterized by:

Link quality, Thequalityofthelink betweenthe twonodes;specifically, theachievabledata transferrateatthenetwork layer, whichdependsonthedistancebetweenthepossibletwo nodes

$\square$ The contactstarting time, Thetimeatwhich the link between thetwo nodesisestablished or alreadyestablishedlink thathasqualitylevelwith newvalue;

$\square$ Acontactending time, thetimeatwhich,thequality leveloflinkhaschangedwhenthelink is removed ordiscarded

The timeintervalbetween any twocontacteventsin thenetwork iscalledframe. Within aframe thenetwork isstatic,i.e., thelink qualitylevelsdonotchangewhichmeansnolink iscreatedor removedornocontacteventisestablished.WedenotethenumberofframesbyF, andthe durationoftheframeis $\mathrm{k}(1 \leq \mathrm{k}$ $\leq$ F)byk;also,allconstantcontacteventsduringeachframek aresaidtobeactivein thatsameframe. Thevehicle shares inthenetwork atframek is represented bya vertex $(1 \leq \mathrm{i} \leq \mathrm{V})$ inthe above graph, whereaseachcandidate AP locationismappedwithineachframekontoavertex $\quad(1 \leq \mathrm{i} \leq \mathrm{A})$. Wedenoteby and.Thesubset of vertices representing the downloader's that existing in the network at frame k denoted.

Withineachframek, adirectededge(

downloadervehicle andanothervehicle

Eachedgeofthisframetypeisassociatedwithaweightw( correspondingcontactevent. Theset havingsuch edges isdenotedas. )existsfromvertextovertexif acontactbetweenthenonisactiveduring thatframe. ), equaltotherateofthat

Similarly,adirectededge ( , )comesfromvertextovertexifacontact between the candidate AP Aiand the vehicle isactive during frame $\mathrm{k}$ These edgesare related withweightsw(, ), equivalenttothecontacteventrate, andtheirsetisdefined as a.A directededge( , ) is alsodrawnfromany 
vertex toanyvertex,for $1 \leq \mathrm{k} \leq \mathrm{F}$. Whiletheedgesin and )modelthepossibilitythatanondownloader vehicle duringitsassociationfromframektoframek +1 .However,dealingwithvehicularnodesas constrainedhand-helddevices, wetaketheweightofsuchedgestobe assumeonaninfini )ofinfiniteweightisheldbetweentwo anyverticesornodesrepresentingthesamecandidateAPattwosuccessiveframes,i.e.,from $(1 \leq \mathrm{k} \leq \mathrm{F})$.Wewillrefertotheedgesofthekind $(, \quad)$ or $($,)asintra-nodal. representtransmissionopportunity,thoseoftheform( physically carries somedata conflictedtoresource-
Adirectededge(

Finally,inordertooriginateamax-flowproblemovertheDNTG,weintroducetwovirtual nodes,denotedas and ,respectivelyrepresentingthesourceanddestinationofthetotalflow ofthegraph.Then, thegraphisfinishedwithinfiniteweightedges( ), fromanyvertexto, $1 \leq \mathrm{k} \leq \mathrm{F}$.

The DNTG is therefore a weighted directed graph, representing the network topology developmentovertime.AnexampleofDNTG isgiveninFig.1,representingoneAPand three vehiclesv1,v2,\&v3, withv1 isconsideredasadownloaderandv2,v3vehiclesareacting as relays.Tominimizethegraphsize, inthe aboveexampleweassumethepossiblenetwork-layer rate $\mathrm{w}$ to beconstantduringthe completelifetime ofa link. Note that the graph allows the capture ofallthedata transferparadigmspreviously discussed.Itispossible toidentify paths inthegraph thatcorrespondto(1).InpathCdirectdownload from theAccesspointtothedownloader.(2).In pathBconnected forwarding through3-hops(frame2)and2-hops(frame5)and(3).InpathA carry-and-forwardthrough the movement intime ofthe relay vehicle v3.

\section{The Max-Flow Problem}

Withtheabovetopologygraph,ournextstep istoformulatetheoptimizationproblem themain goal of this problem is to maximize the flow from to, i.e., the total amount of data downloadedbythedownloader's.Denotedbyx ( , )thetrafficflowoveranedgeconnecting two genericvertices, ourintentioncanbe expressedas:

\section{$\sum \sum$}

The max-flow problemneeds to be solved takinginto account several constraints such as, maximum numberofAPsthatcanbeactivated,channelaccess,nonnegativeflowandflow conservation. We detailsuch constraints below.

\section{A. Constraints}

Non-negative flowand flowconservation:theflow oneachexistingedgeinDNTGmustbe greaterthanorequaltozero.Also,foranyvertexinthegraph, theamountofingoingflowinto the vertex mustequalthe amountofoutgoing flow.

Channelaccess:Inviewofthefactthatweconsider

anIEEE802.11-basedMAC

schemewith RTS/CTSandweassumeunicasttransmissions,twoormoreofthefollowing eventscannottake placesimultaneously fora taggedvehicle, and thetimedurationofeachframemustbeshared amongthe tagged vehicle:

1) The vehicle transmits to a neighboring vehicle;

2)Aneighboring vehicle receivesfromanyrelay;

3)The vehicle receivesfroma neighboringrelay;

4)Aneighboringrelaytransmits toany vehicle;

5)The vehicle receivesfroma neighboringAP;

6)AneighboringAP transmits to any vehicle.

The aboveconstraintsallowavehicleunderAPcoverageareatoutilizeeitherI2VorV2V communication within the sameframe. Next, we considera vehicle under thecoverage ofeither oneAPisnotconnected towork inadhocmode, whichmeansthecommunicationwithother vehicleisnotpossible. Then,foreveryframekand,suchthat( , ), the followingconstraintholds: 


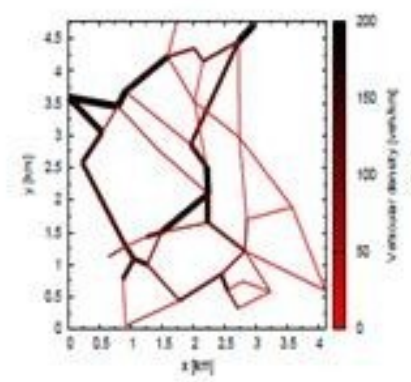

(a) Vebicular density over space

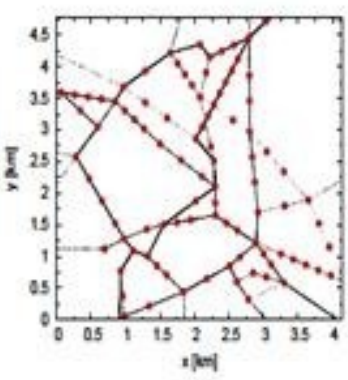

(b) AP candidate locations

Figure2.Simulationscenario:(a)roadlayoutandaveragedensityof vehiclescomputedoverawholeday; (b) givingoutoftheAPcandidatelocationsovertheroadlayout.

$$
\mathrm{x}()(\mathrm{C},)
$$

Where, $\mathrm{i}=1 \ldots$ A,areBooleanvariables, IfthecandidateAPAiisactivatedthevalue island the value becomes0 otherwise.

MaximumnumberofactiveAPs:The finalsetof constraintsimposes thatnomorethanone candidate APs areselected, through the variablesyi.Then, foranyi, wecan write:

\section{$\sum$}

Where M Risa randomly large positive constant.

\section{Deriving Design Guidlines}

Weinfluencetheproblem formulationobtainedintheprevioussection concernthemostin contentdownloadingprocessinvehicularnetworksand toillustratewhichfactors toproviderealistic

hintsforthedesignofasystem. Weconsiderareal-worldroadtopology, coveringanareaof10km2intheurbanarea. Thevehicularmobility trace intheregionhasbeensynthetically generated aturbanarea, throughamultiagentmicroscopic trafficsimulator.InFig.2(a), wedescribethe roadlayoutwhich explainsthe differenttraffic methodologyobserved overeach road layout.

WeconsideratraditionalVANETtechnologypenetrationrate, whichmeansthatonlyafraction of the vehiclesinthenetwork,namely 20\%,isequippedwith acommunication interfaceor communicationdeviceand isready toparticipateinthecontentdownloading process, eitheras relaysorasdownloader's. Also,thenumberofvehiculardownloader's thatconcurrently request contentisassumedtobe $1 \%$ ofthevehiclesparticipatinginthenetwork.APlocationsare selectedalong theroadssuchthat the distance betweentwoadjacentAPs isslightlyequal to150 m,resulting in92candidatelocations, shown inFig.2(b).Thevalueoftheachievablenetwork- layerrate betweenany two nodes isattunedaccording tothedistance betweenthem. Webounded themaximumnodetransmissionrangeto200m;thisdistanceallowstheestablishmentofa reliablecommunicationin $80 \%$ of the cases.

Sincewe make use ofarealistic mobilitymodel, ineach roadtopologythe intensityof the vehiculartraffic variesdepending ontheroadsegmentand time period ofthe day. In Fig. 3a, 3b, and 3c, wedescribetheroad layoutofthe urban, village, and suburbanvillageenvironments, stressthedifferenttrafficvolumesobserved overeachroadsegment:Thicker,highlighted density segmentsidentify theroadslayoutcharacterizedby highervehiculardensity.Asfarasvehiculartraffic variations ingivenroad layoutisconcerned,weconsideronly timeperiodscorresponding tothe densityofvehicles.

Intheurban, village, andsuburbanroadlayouttraces, eachenduringabout6hours, thisleadsto

anaveragedensityof 90, 62.5 and 33.5 vehkm, respectively. The value ofthe attainable networklayerratebetweenevery twonodesisadjustedaccording tothedistancebetweenthem. To thisend,we refer to the 802.11aexperimental results thatobtain thevaluesshowninFig.3d, andweuse them assamplesof theachievablenetwork-layerrate.Note thatweendsupthe maximum nodetransmissionrangeto $200 \mathrm{~m}$, because, thisdistanceallows theestablishmentofa reliablecommunicationin 80 percentofthecases.

GiventhatA^locationshavetobeactivated,theresultofthemax-flowprobleminSec. 4 theAPdeploymentthatmaximizestheaggregatedownloadthroughput. Webenchmark the the performance ofouroptimalMax-flow strategyagainstthefollowingAP deploymentpolicies: 
Random:Accordingtoauniform distribution,AP^locationsarerandomly selectedamongthe candidate.

Crowded:Itselectsthe $\mathrm{AP}^{\wedge} l o c a t i o n s w h o s e ~ c o v e r a g e a r e a e x h i b i t s$, above thehighestvehicular density;

Contact:Itpicksupthe AP^locationsthatmaximizetheadditionofthecontactopportunities between vehiclesand APs.

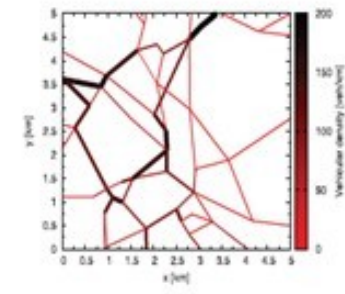

(a) Urban: traffic over space

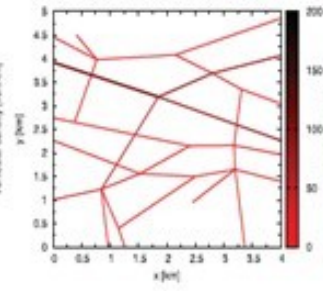

(b) Village: traffic over space

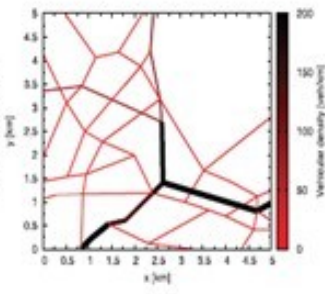

(c) Suburban: traffic ower space

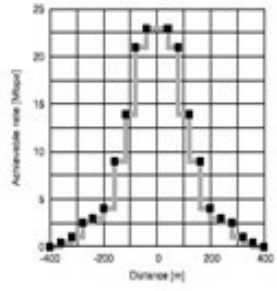

(d) Network-layer rate

Figure3.Roadlayoutinthe(a) urban,(b) village,and(c) suburbanscenarios,andachievablenetwork-layer ratecharacterizationasa functionofdistance(d).

Particularly,foreachvehicle, thecontactopportunity isexpressedas thefractionof theroad section lengths traveledwhile undercoverageofat leastoneAP.Once the active APlocationsin thegivenroadlayoutaredeterminedaccording toany oneoftheabovethreepolicies, they are usedinthemaxflowproblemformulationtosecurethevaluesofthebinaryvariablesyi.Since thesystem throughputisobtainedastheresultofthemax-flowproblem withthepreferredAP locationsyi, the resultsweshowrepresent the preeminentperformanceone canachievewith each deploymentstrategy. Fig. 3 shows theaveragecontentdownloader throughput fordifferent deploymentstrategies, withthe function ofthenumberofactive APs A^.

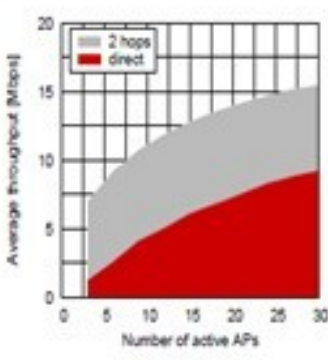

(a) 2-hop limit

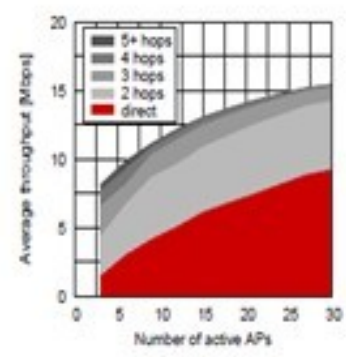

(b) unlimited

Figure4.PartitionoftheaveragedownloaderthroughputwithrespecttothenumberofrelaysbetweenAP anddownloader(Max-flowdeploymentstrategy)

Inordertodemonstratethe absolute resultof the throughputfigures reported above,wefocuson the Maxflowdeploymentstrategy andlook at the numberofhops thatdatagothroughbefore reaching theirdestination.InFig.4(a), thehoplimitisset to2whichmeansthenumberofrelays betweenAPsis2, thustheplot intheabovegraphdescribestheportionof

downloaderthroughputisduetodirectdatatransfersandwhichisinsteadreachedusingone

relay,whenthenumberofdeployedAPs issmallthen thelasthop largely dominatesthe previous hop.AstheexistenceofAPsbecomesmorepervasive,directtransfersparadigmareclearly more frequent.However, it ismostimportanttoobservethattheamountofdatadownloadedthrough one relayremainsconstant,even when $25 \mathrm{APs}$ covering $50 \%$ ofthe roadlayoutare deployed. The proportionofthroughputachievedthroughdirectand multihopdatatransfersdoesnotchange whentheboundaryonthenumberofallowedhopsisremoved,inFig.4(b). Finally,

the comparisonbetween Fig.4(a)andFig.4(b)shows thecomplexity due to theuseofmore than onerelayata timecanbeeliminatedwithoutsignificantdestruction. Tosummarize, weillustrate thefollowingconclusions:

$\square$ Traffic relaying, through eitherconnected forwarding or carry-and-forward, can considerably increasetheaverageper-downloader throughput,evenwhenthe roadlayoutis covered bymoreAPs;

$\square$ Multi-hopdatatransfersinvolvingmorethanonerelayarelessbeneficialtothecontent downloadingprocess. 


\section{Vehicle Density Based Access Point Data Downloading}

In addition, the access point or relay tracks the vehicle capability prior and sends the corresponding lowqualityorhighquality file.Thisachievesthevehicle toreceivetheproperfile resource.

Vehicledensityiscalculatedbasedonprevioustemporalchangesandthenewvehicledensityiscalculated. Theaccesspoints'capabilitiesareadjustedso thatitworksmore inhighvehicle densityenvironmentand works less inlowvehicledensityenvironment.

\section{Security Issues}

\subsection{Digital signatures as a buildingblock}

Themessage authenticityisnecessarytoprotectVANETs

fromoutsiders.Butsince safety messageswillnotcontainany sensitiveinformationconfidentiality isnotrequired.Inthissystem, theexchangeofsafety messagesby vehiclesinaVANETneedsauthenticationofmessagebutno needforencryptionofsuchmessage.Symmetricauthenticationmechanismsusually encourage lessoverhead per message than their asymmetric counterparts. In the VANETsetting,safety messagesaretypicallystandaloneandshouldbesenttoreceiversasquickaspossiblesothe

digitalsignaturesareabetterchoice.Infact, aprefacehandshake isnotsuitableandactually creates moreoverhead. In addition, with the huge amount of network participants and the irregularconnectivitytoauthenticationservers,aPKI(PublicKeyInfrastructure)mechanismis themostsuitable methodfor implementation ofmessageauthentication.

\subsection{Estimationofthesignaturesize}

AsweintendusingaPKI

forsupportingsecurityinVANETs, itissignificanttochooseaPublic KeyCryptosystem (PKCS)withatolerableimplementationoverhead inthevehicularnetwork. According toDSRC,safetymessagesaresentwithaperiodicity of 100 to $300 \mathrm{~ms}$.thisinflictan upperbound on the processingtime overhead;thisoverheadisshown below:

$T_{\text {oh }}(M)=T_{\text {sign }}(M)+T_{t x}\left(M \mid \operatorname{Sig}_{\text {PrkV }}[M]\right)+T_{\text {veriff }}(M)$

Where $T_{\operatorname{sign}}(M), T_{t x}(M)$,and $T_{\text {verify }}(M)$ arethenecessarytimedurationstosign, transmit,and verifyamessageM,respectively;Sig ${ }_{\mathrm{r} r \mathrm{k}}[\mathrm{M}]$ isthesignatureofMandalsoincludestheCA's

Certificateof thesigning keyby thesending vehicleV.Theaboveexpressionshowsthethree factorsthataffect thechoiceofaparticularPKCS: (1) theexecutionspeedsofthesignature generation(2)theverification operations, and(3) the sizesofkey, signature, and certificate.

Since theactualsizeofencryptedmessagesisbetween 100 and200bytes, beforebeing signing, the message ishashed.Theoverheadis almost constantfora given cryptosystem.

Hence, it ispossibletoevaluate differentoptionsat leastrelatively toeachother.Infact, thereare morenumberofcandidatePKCSforimplementingthePKI inaVANET.Toensurethefuture securityof thePKCS, andtakinginto accountthe deploymentscheduleofDSRC.

Table1:SizeandtransmissiontimeofPKCS

\begin{tabular}{|l|c|l|}
\hline PKCS & Sigsize(bytes) & Ttx(Sig)(Ms) \\
\hline RSA & 256 & 0.171 \\
\hline ECDSA & 28 & 0.019 \\
\hline NTRU & 197 & 0.131 \\
\hline
\end{tabular}

Table2:Comparisonofsignaturegenerationandverificationtimesonamemory-constrainedPentiumII 400MHzworkstation

\begin{tabular}{|l|c|c|}
\hline PKCS & Generation(ms) & Verification(ms) \\
\hline ECDSA & 3.255 & 7.617 \\
\hline NTRU & 1.587 & 1.488 \\
\hline
\end{tabular}

Welistrecordsforpublic keyand signaturesizes: 
1. RSASign: the keysizeandsignature sizesarelarge(256bytes).

2. ECC(EllipticCurveCryptography):itissmallerthanRSA(28bytes),slowerin verificationbutfasterin signing.

3. NTRUSign 4 :thekeysizeisliesbetweentheRSAandECC(197bytes),butinboth signingand verification.itis much fasterthanthe RSAand ECC

InDSRC theleastdatarateis $6 \mathrm{Mbps}$ (forsafety messaging datarateistypically 12Mbps), the transmissionoverhead(at12Mbps)isacceptable, andthesetwooptionsareshowninTable1 andTable2givesapproximateexecutiontimesofsignature generationandverificationfor (EllipticCurveDigitalSignatureAlgorithm)andNTRUSign. Thesefiguresin the table takenonlyassuggestivefor thespecificplatformsuchasPentiumII $400 \mathrm{MHz}$ with memoryconstraints.

ECDSA shouldbe

Inconclusion,wecanobservethatintermsofperformance,ECDSAandNTRUoutperform

RSA.Comparedtoeachother, theadvantageofECDSA isitssmallandeconomically whereasNTRU'sismorespeed thanECDSA. Theresultshoulddependoncase-specific evaluations.

designed;

\section{Conclusion}

Weexamined themain factorsaffecting theperformanceofcontentdownloadingprocessin vehicularnetworks,by formulating andsolving amax-flowproblem overatimeextendedgraph representinga realistic vehiculartrace.

Theimportantresults in oursystemare asfollows:

$\square$ Ourmajorideasarethatadensity-basedAPdeploymentyieldsperformanceclosetothe optimum result, and that multi-hop traffic delivery is valuable, although the gain is negligiblebeyond 2 hops fromthe AP.

$\square$ Theaccesspoints'capabilitiesareadjustedsothatitworksmoreinhighvehicledensity environmentandworks less inlowvehicle densityenvironment.

To ourbestknowledge,thispaperaddressingthesecurity ofvehicularnetworksinaefficientand quantified way.

Intermsoffuturework,weaim tofurtherdevelop thisproposal.Inparticular,weplantoexplore inmoredetail the respectivemeritsofkeydistributionby themanufacturersorby legislative bodies;we willalsogoingtocarryoutadditionalnumericalevaluations ofthe solutions.

\section{References}

[1]. M.Francesco.C. Claudio, C. Carla-Fabianaand F.Marco,"Optimalcontentdownloadinginvehicular networks,” proc.IEEEINFOCOM,July2013.

[2]. U.Paul,A.P.Subramanian,S.R.DasandM.M.Buddhikot,“ Understanding Traffic Dynamicsi Cellular Data Networks,”Proc. IEEEINFOCOM,Apr.2011.

[3]. K.Pentikousis,M.Palola,M.Jurvansuu,andP.Perl,“Activegoodputmeasurementsfromapublic G/UMT Snetwork, ”IEEECommunicationsLetters,vol. 9, pp.802-804,2005.

[4]. P.Reichl,M.Umlauft, J.Fabini,R.Lauster,andG.Pospischil,"Project WISQY:Ameasurement- basedend-to-endapplication-level performancecomparisonof2.5Gand3Gnetworks,"inProc. Fourth Ann. Wireless Telecomm. Symp(FTS),2005.

[5]. K.Mattar,A.Sridharan,H.Zang,I.Matta, andA.Bestavros, "TCPoverCDMA2000networks:A

crosslayermeasurementstudy,"inProc.PAM, 2007

[6]. DWillkomm, S. Machiraju,J.Bolot,andA.Wolisz,"Primaryusersincellularnetworks:Alarge-scale mea surement study,"inProc.DySPAN,2008.

[7]. R.Keralapura, A.Nucci,Z.-L.Zhang,andL.Gao,"Profilingusersina3Gnetworkusinghourglas co-clusteringn, "in Proc. ACMMobiCom,2010.

[8]. VeriWiseAssetIntelligence.http://www.ge.com/equipmentservices/asseintelligence/.

[9]. A.Balasubramanian,R.Mahajan, A.Venkataramani,B.N.Levine, andJ.Zahorjan.InteractiveWi-Fi Connectivity for MovingVehicles.InProc.ofACMSIGCOMM,Sept.2008.

[10]. V.Bychkovsky,B.Hull,A.K.Miu,H.Balakrishnan, andS.Madden. A Measurement Study of $\begin{array}{lcccc}\text { Vehicular Internet AccessUsingInSituWi-FiNetworks.InProc.o } & \text { f } & \text { ACMMOBICOM,Sept.2006. } \\ \text { J.Eriksson,H.Balakrishnan, andS.Madden.Cabernet:AWi-Fi-Based } & \text { Vehicular } & \text { Content }\end{array}$ Network.InProc.ofACMMOBICOM,Sept.2008.

[11]. V.Navda,A.P.Subramanian,K.Dhanasekaran,A.Timm-giel,andS.R.Das.MobiSteer:Using Steerable Beam Directional Antenna for Vehicular Network Access .In Proc.o fMOBISYS, 2007. J.OttandD. Kutscher. Drive-thru Internet: IEEE 802.11 bfor"A utomobile" Users. In Proc.ofI NFOCOM, Mar.2004.

[12]. Z.ZhengS.Kumar,andP.Sinha,."Alpha Coverage:BoundingtheInterconnectionGapforVehicular Internet Access.” Technical ReportOSU-CISRC-1/09-TR03,ftp://ftp.cse.ohio-state.edu/pub/tech-report /2009/ TR03. pdf,2009.

[13]. Z.Zheng,Z.LuandP.Sinha,"Maximizing theContactOpportunityforVehicularInternetAccess,” Proc. IEEEINFO- COM, Mar.2010

[14]. GoogleWi-Fi.Google'sMountainViewWi-FiNetwork.http://wifi.google.com/. 
[15]. TakingWirelesstotheMax.BusinessWeek(business-week.com/go/techmaven) ,pages101-102, Nov.2008.

[16]. WiMax.comFAQ.http://www.wimax.com/education/faq/,2008

[17]. U.S.CensusBureau-TIGER/Linehttp://www.census.gov/geo/www/tiger/

[18]. VeriWiseAssetIntelligence.http://www.ge.com/equipmentservices/asseintelligence/.

[19]. M.FioreandJ.M.Barcelo-

Ordinas,“CooperativeDownloadinUrbanVehicularNetworks,”Proc.IEEESixthInt'lConf.MobileAdHocandSensorSystems(MASS),O ct.2009

[20]. F.Aidouni,C.Magnien,M.Latap,“TenweeksinthelifeofaneDonkeyserver”,Hot-P2P’09, Rome,Italy,May2009.

[21]. K.Fall,“Adelay-tolerantnetworkarchitectureforchallengedInternets”,Proc.ACMSigcomm'03,Karlsruhe, Germany,August2003.

[22]. S.Keshav, D.Hadaller,S.Agarwal,andT.Brecht,"VehicularOpportunisticCommunicationunder the Microscope, "Proc. ACMMobiSys,June2007 